Bull. Austral. Math. Soc.

VOL. $61(2000) \quad[85-88]$

\title{
ON CONVEX VECTOR OPTIMISATION
}

\section{JOYDEEP DUTTA}

In this article we present a simple method to deduce necessary conditions for weak minimisation of a convex vector program in a Banach space. Our main tool here will be the generalised Jabcobian of Ralph.

\section{INTRODUCTION}

Let $X, Y$ and $Z$ be Banach spaces. Let $K \subseteq Y$ and $S \subseteq Z$ be closed and convex cones in $Y$ and $Z$ respectively. Here we shall be concerned with the following vector optimisation problem (VP).

$$
\begin{gathered}
\text { Weak Minimise } f(x) \\
\text { subject to }-g(x) \in S
\end{gathered}
$$

where $f: X \rightarrow Y$ and $g: X \rightarrow Z$. We use $Y^{\prime}$ and $Z^{\prime}$ to denote the dual spaces of $Y$ and $Z$ respectively. We shall assume throughout that int $K \neq \emptyset$ and int $S \neq \emptyset$. We shall denote by $K^{*}$ and $Z^{*}$ the dual cones of $K$ and $S$ respectively. A point $x^{*} \in X$ is said to be weak minimum for (VP) if it is feasible for (VP) and there exists no feasible $x$ such that $f(x)-f\left(x^{*}\right) \in-$ int $K$. For any given $K$-convex function $h: X \rightarrow Y$, the vector subdifferential of $h$ at $a \in X$ is given by

$$
\partial h(a)=\{A \in L(X, Y): h(x)-h(a)-A(x-a) \in K \text { for all } x \in X\} .
$$

Our aim is to deduce necessary KKT conditions for weak minimisation of (VP) in terms of the subdifferentials of $f$ and $g$. In this context it is important to know when $\partial h(a)$ is non-empty since in general the vector subdifferential of a $K$-convex function could be empty. Under mild conditions one can assert its non-emptiness by using the idea of Ralph's generalised Jacobian [4] defined for locally Lipschitz vector functions. Given $h: X \rightarrow Y$ a locally Lipschitz vector function, the Ralph generalised Jacobian at $a \in X$ is defined by

$$
\partial^{R} h(a)=\left\{A \in L(X, Y): \forall(u, \lambda) \in X \times Y^{\prime}, \quad(\lambda h)^{\circ}(a, u) \geqslant \lambda A u\right\}
$$

Received 15th April, 1999

This work was done when the author was visiting the Indian Statistical Institute, New Delhi. The author is deeply indebted to Professor B.D. Craven for his help and suggestions to improve an earlier version of this paper.

Copyright Clearance Centre, Inc. Serial-fee code: $0004-9727 / 00 \$ A 2.00+0.00$. 
where $(\lambda h)^{\circ}(a, u)$ denotes the Clark generalised directional derivative [1] of the scalar locally Lipschitz function $\lambda h$ at the point $a \in X$ and in the direction $u$. It is known from [4] that when $Y$ is a reflexive Banach space then $\partial^{R} h(a)$ is a non-empty and weak operator compact subset of $L(X, Y)$. Though in general the Ralph's generalised Jacobian is different from the Clarke's generalised Jacobian [1]; the Ralph's generalised Jacobian coincides with the Clarke subdifferential when $Y=R$. (See [4].) Again when $Y$ is reflexive, from Ralph [5] we have that for all $\lambda \in Y^{\prime}$ the subdifferential $\partial^{C}(\lambda h)(a)=\lambda \partial^{R} h(a)$, where $\partial^{C}(\lambda h)(a)$ represents the Clarke subdifferential [1] of the scalar function $\lambda h: X \rightarrow R$ at the point $a \in X$. For more details on the Ralph subdifferential see $[4]$ and $[5]$.

\section{MAIN ReSUlts}

We begin this section by proving the following lemma.

Lemma 2.1. Let $f: X \rightarrow Y$ be a continuous $K$-convex function which is also locally Lipschitz. Assume that $Y$ is a reflexive Banach space. Then $\partial^{R} f(a) \subseteq \partial f(a)$ for all $a \in X$.

PROOF: Since $Y$ is reflexive we know that [4] $\partial^{R} f(a)$ is nonempty for all $a \in X$. Consider any $A \in \partial^{R} f(a)$. Then by the definition of the Ralph generalised Jacobian at $a \in X$ we have that

$$
\text { for all }(u, \lambda) \in X \times Y^{\prime} \text { we have }(\lambda f)^{\circ}(a, u) \geqslant \lambda A u \text {. }
$$

Since $K^{*} \subseteq Y^{\prime}$ we have that

$$
\text { for all }(u, \lambda) \in\left(X \times K^{*}\right),(\lambda f)^{\circ}(a, u) \geqslant \lambda A u \text {. }
$$

Again it is well known that for any $\lambda \in K^{*}$ the function $\lambda f: X \rightarrow R$ is a Lipschtiz convex function since $f$ is a locally Lipschitz $K$-convex function. Hence the Clarke generalised derivative $(\lambda f)^{\circ}(a, u)$ coincides with the one sided directional derivative $(\lambda f)^{\prime}(a, u)$ of the convex function $\lambda f$. This fact along with the convexity of $\lambda f$ shows that

$$
\lambda f(x)-\lambda f(a) \geqslant(\lambda f)^{\prime}(a, x-a) \geqslant \lambda A(x-a) \text { for all } x \in X .
$$

Hence we have that

$$
\lambda f(x)-\lambda f(a)-\lambda A(x-a) \geqslant 0
$$

for all $x \in X$ and for any $\lambda \in K^{*}$. By a standard separation argument we have that

$$
f(x)-f(a)-A(x-a) \in K \text { for all } x \in X \text {. }
$$

this proves the lemma. 
REMARKS. The above result additionally shows the conditions under which one can guarantee the nonemptiness of the convex vector subdifferential. In fact without assuming $f$ to be a vector Lipschitz function we could have equivalently assumed that the closed and convex cone $K$ is normal. In fact if $Y=R^{m}$ and $K=R_{+}^{m}$ then the subgradient of the vector convex function can be constructed from the subgradient of the component scalar functions. Let us mention that if $f$ is Frechet differentiable at $a \in R^{n}$ then the Frechet derivative $d f(a) \in \partial f(a)$. Moreover if $K$ is pointed then $\partial f(a)=\{d f(a)\}$. Also note that the vector subdifferential $\partial f(a)$ is a closed and convex subset in $L(X, Y)$.

In the context of the non-emptiness of the vector subdifferential in Banach spaces it is important to note the work due to Craven and Glover [3] where they have used a different approach to prove the non-emptiness of the vector subdifferential of a convex vector function. One of the major assumptions considered in Craven and Glover [3, Theorem 3.1] is the fact that the function is densely Gateaux differentiable in the sense that the set of non-Gateaux differentiability forms a thin set. Craven and Glover define a vector subdifferential and then show that the convex vector subdifferential contains the vector subdifferential they define. For more details see Craven and Glover [3]. Our approach differs from that of Craven and Glover in that it does not require the notion of dense Gateaux differentiability in the sense mentioned above. It is also interesting to note that [3, Theorem 3.1] can be deduced from [3, Lemma 4] and the above lemma.

TheOREM 2.1. Consider the vector program (VP), in which $Y$ and $Z$ are reflexive Banach spaces, $K \subseteq Y$ and $S \subseteq Z$ are closed convex cones with interior,

$$
\begin{aligned}
& f: X \rightarrow Y \text { is locally Lipschitz and } K \text {-convex, } \\
& g: X \rightarrow Y \text { is locally Lipschitz and } S \text {-convex. }
\end{aligned}
$$

Let $x^{*} \in X$ be a weak minimum for (VP). Then there exist $(0,0) \neq(\tau, \lambda) \in K^{*} \times S^{*}$ such that

(i) $\quad 0 \in \tau \partial f\left(x^{*}\right)+\lambda \partial G\left(x^{*}\right)$

(ii) $\lambda g\left(x^{*}\right)=0$.

Proof: Since $Y$ and $Z$ are reflexive Banach spaces it is clear that $\partial^{R} f\left(x^{*}\right)$ and $\partial^{R} g\left(x^{*}\right)$ are nonempty. Since $x^{*}$ is a weak minimum the following system

$$
-\left(f(x)-f\left(x^{*}\right), g(x)\right) \in \operatorname{int}\left(K^{*} \times S^{*}\right), \quad x \in X
$$

has no solutions. By using Craven's Basic Alternative Theorem (see [2, Theorem 2.1]) it can be shown that there exists $(0,0) \neq(\tau, \lambda) \in\left(K^{*} \times S^{*}\right)$ such that $x^{*}$ minimises the scalar Lagrangian function $L(., \tau, \lambda)$ where $L(x, \tau, \lambda)=\tau f(x)+\lambda g(x)$ and additionally 
one can deduce that $\lambda g\left(x^{*}\right)=0$. Again as $f$ and $g$ are locally Lipschitz, $\tau f+\lambda g$ is also locally Lipschitz and hence from Clark [1] we have that

$$
0 \in \partial^{C}(\tau f+\lambda g)\left(x^{*}\right) \text { and this implies that } 0 \in \partial^{C}(\tau f)\left(x^{*}\right)+\partial^{C}(\lambda g)\left(x^{*}\right) .
$$

Again we know that $\partial^{C}(\tau f)\left(x^{*}\right)=\tau \partial^{R} f\left(x^{*}\right)$ and $\partial^{C}(\lambda g)\left(x^{*}\right)=\lambda \partial^{R} g\left(x^{*}\right)$. This shows that $0 \in \tau \partial^{R} f\left(x^{*}\right)+\lambda \partial^{R} g\left(x^{*}\right)$ and now by using Lemma 2.1 the theorem is proved.

REMARKS. If we assume some constraint qualification then one can easily show that $\tau$ is non-zero and one can normalise $\tau$ by considering it to be 1 . This would lead to the Karush-Kuhn-Tucker conditions. The KKT conditions are also sufficient since $f$ and $g$ are $K$-convex and $S$-convex functions respectively. One can now construct the Wolfe dual easily and prove the strong duality theorem.

\section{REFERENCES}

[1] F.H. Clarke, Optimization and nonsmooth analysis, Canadian Mathematical Scoeity Series of Monographs and Advanced Texts (J. Wiley and Sons, New York, 1983).

[2] B.D. Craven, Mathematical programming and control theory (Chapman and Hall, London, 1978).

[3] B.D. Craven and B.M. Glover, 'An approach to vector subdifferential', Optimization 38 (1996), 237-251.

[4] B.M. Glover and D. Ralph, 'First order approximations to nonsmooth mappings with application to metric regularity', Numer. Funct. Anal. Optim. 15 (1994), 599-620.

[5] D. Ralph, Rank-1 support functionals and generalized Jacobian, piecewise linear homemorphisms, (Ph.D. Thesis) (University of Wisconsin, Madison, 1990).

Department of Mathematics

P.D. Womens College

Jalpaiguri 735101

West Bengal

India 